\title{
OpenSky Report 2016: Facts and Figures on SSR Mode S and ADS-B Usage
}

\author{
Matthias Schäfer*, Martin Strohmeier ${ }^{\dagger}$, Matthew Smith ${ }^{\dagger}$, Markus Fuchs ${ }^{\ddagger}$, \\ Rui Pinheiro ${ }^{\S}$, Vincent Lenders ", Ivan Martinovic ${ }^{\dagger}$ \\ *University of Kaiserslautern, Germany \\ schaefer@cs.uni-kl.de \\ ${ }^{\dagger}$ University of Oxford, UK $\quad{ }^{\ddagger}$ SeRo Systems, Germany \\ $\S$ University of Hagen, Germany \\ pinheiro@opensky-network.org \\ ף armasuisse, Switzerland \\ vincent.lenders@armasuisse.ch
}

\begin{abstract}
This paper provides up-to-date statistics on SSR Mode $S$ and 1090ES ADS-B usage from the OpenSky Network. By analyzing the large host of real-world data collected by OpenSky, we provide insights on the current composition of Mode $S$ downlink communications, aircraft equipage, interrogation patterns and much more. We publish up-to-date, empirically validated numbers on the status of the ongoing ADS-B deployment based on data collected over large areas in Europe, North America, and New Zealand, and categorize these aircraft by incorporating publicly available data sources.
\end{abstract}

\section{INTRODUCTION}

Mode $\mathrm{S}$ is one of the most important technologies in air traffic management as it supports the operation of secundary surveillance radar (SSR), traffic alert and collision avoidance systems (TCAS), and the Automatic Dependent Surveillance - Broadcast (ADS-B) surveillance. In SSR Mode S, transponders in aircraft are selectively interrogated by sensors (radars) to perform ranging and bearing as well as to provide enhanced situational awareness with the exchange of binary-encoded surveillance information. TCAS systems also rely on the the Mode $\mathrm{S}$ data link to detect collision hazards and exchange resolution advisories among each other. In ADS-B, the new standard that will become mandatory in most airspaces by 2020, Mode $S$ transponders elicit the periodic or event-driven transmission of extended squitters (ADS-B messages) in order to provide position, velocity and identity reports to aircraft and ground stations in range.

While Mode $\mathrm{S}$ has been operational for years in many airspaces around the world, we are not aware of any larger measurements efforts that aim at characterizing how Mode $\mathrm{S}$ is actually used in the wild. To fill this gap, this work analyzes a large host of real-world data collected by the OpenSky Network 1 The OpenSky Network is a large-scale sensor network with crowdsourced Mode $S$ receivers that has been collecting air traffic communication data for over three years. The sensors in OpenSky are operated continuously on the ground by volunteers in a crowdsourcing manner. As of this writing, the sensor network comprises more than 50 active sensors deployed over large areas in Europe, North America, and New Zealand. The total amount of Mode $S$ data collected

\footnotetext{
${ }^{1}$ http://opensky-network.org
}

\begin{tabular}{|l|l|l|}
\hline DF & Information & Application \\
\hline \hline 0 & altitude & collision avoidance \\
\hline 4 & altitude & surveillance \\
\hline 5 & squawk & surveillance \\
\hline 11 & transponder address & surveillance \\
\hline 16 & altitude, threat resolution & collision avoidance \\
\hline 17 & ADS-B message & surveillance \\
\hline 18 & ADS-B/TIS-B message & surveillance \\
\hline 19 & Military applications & - \\
\hline 20 & altitude, Comm-B & surveillance \\
\hline 21 & squawk, Comm-B & surveillance \\
\hline 24 & ELM & data transfer \\
\hline
\end{tabular}

Table I: Mode S downlink formats, the contained information, and their application.

so far comprises 150 billions messages from more than 80,000 transponders.

Based on this rich source of data, we provide insights on tracking methods, the composition of Mode S downlink communications, aircraft equipage, interrogation patterns and much more. To the best of our knowledge, we are also the first to publish up-to-date numbers on the status of the 1090ES ADS-B deployment based on empirical Mode S data.

\section{About Mode S AND ADS-B}

This section provides background information on the technology, operation, and deployement of Mode S and ADS-B.

\section{A. Provided Information and Downlink Formats}

The reception of Mode $S$ signals requires a line of sight to the transmitter. Since OpenSky's ground-based receivers usually do not have a line of sight connection to the interrogators and since interrogations are done on a different frequency $(1030 \mathrm{MHz})$, OpenSky only collects messages that are sent over the Mode S downlink on the $1090 \mathrm{MHz}$ channel (transponder to interrogator). Different messages types, called downlink formats (DF), are defined in the Mode $\mathrm{S}$ specification [1]. An overview of the downlink formats is provided in Table II. The formats have been coarsely classified by their applications.

The first application is surveillance for air traffic control through ground sensors. Aircraft are first acquired using all- 
call replies (DF 11). Once acquired, the ground sensor can interrogate altitude (DF 4, 20), transponder codes called squawks (DF 5, 21), and extended surveillance data from avionics such as intent- and status information via Comm-B messages (DF 20, 21). Squawks are assigned to the flight by the responsible controller mainly for identification purposes, but they are also used by pilots to indicate emergencies, radio failure, and even aircraft hijacking.

The second application is collision avoidance (known as airborne collision avoidance system, or ACAS). Naturally, these messages are exchanged between aircraft (air-to-air) rather than between aircraft and ground sensors (air-to-ground). They contain altitude information (DF 0 and 16) as well as additional information exchanged by aircraft to resolve potential threats (DF 16).

Although DFs 17 and 18 can also be attributed to the surveillance application, they differ from the others in several ways. The most significant difference is that these messages are not transmitted upon interrogation. Transponders broadcast these formats automatically ("squitter") and they can contain all information needed to determine the aircraft's threedimensional location, velocity, identity, or operational status. Thus, their use is not limited to air traffic surveillance, these information can also be used by other aircraft for collision avoidance. These squittered information are called the Automatic Dependent Surveillance-Broadcast (ADS-B).

The third application is general data exchange. Over the Comm-D data link (DF 24), ground sensors can retrieve up to 280 bits, fragmented over multiple extended length messages which offer an 80 bit data field each.

\section{B. Operation}

Mode $\mathrm{S}$ downlink transmissions are elicited in two different ways: interrogation-based (SSR/ACAS) and non-solicited messages (ADS-B/ACAS).

The interrogation-based operation in SSR is depicted in Figure 1. A ground sensor is using a rotating directional antenna to send interrogation signals to aircraft with interrogations being done in two phases, all-call and roll-call $\left.\right|^{2}$ In the all-call phase, the interrogator broadcasts all-call requests to all aircraft, which then respond with an all-call reply. By measuring the time between transmission of the request and reception of the reply, the interrogator estimates the distance to the aircraft (ranging). As soon as an aircraft enters the interrogator's operational range, it is added to the interrogator's list of acquired aircraft. Once an aircraft has been acquired, the ground sensor starts selectively requesting surveillance information from the aircraft during the so called roll-call phase of an interrogation. In order to reduce the interference on the communication frequency, the interrogator instructs the aircraft to ignore subsequent all-call requests for the duration of the acquisition, i.e., until it leaves the operational range. This is referred to as the lockout protocol.

\footnotetext{
${ }^{2}$ Ground sensors may also use omni-directional antennas as in the case of multilateration. However, as this work focuses on the classic SSR Mode S, we omit multilateration in our considerations.
}

As opposed to SSR, ADS-B is based on non-solicited transmissions. Transponders broadcast extended squitter (DF 17 and 18) periodically or event-based. For example, position and velocity messages are both broadcast twice per second and emergency messages are only broadcast if needed. ADS$\mathrm{B}$ messages are received by ground stations or other aircraft.

Collision avoidance systems use both kinds of transmissions. On the one hand, transponders elicit the automatic transmission of all-call replies (DF 11) periodically to be detected by nearby aircraft. On the other hand, they can also interrogate other aircraft to obtain more frequent updates (DF $0,16)$ for the purpose of threat detection and resolution.

\section{Addressing}

To be able to selectively interrogate aircraft, transponders on-board aircraft have been assigned a unique 24-bit identifier. The range of this identifier is divided into blocks by the ICAO and each block is reserved for a country [3]. The assignment of addresses from a block to transponders is done by the respective national authority where the aircraft is registered. We refer to these identifiers as ICAO 24-bit address. They are also included in all Mode $\mathrm{S}$ replies and ADS-B messages and allow identifying the sender and its origin country.

Interrogators are assigned one of 80 possible interrogator codes which are included in all-call requests. These interrogators codes are not globally unique and the same codes may be assigned to different interrogators at different locations. Aircraft distinguish interrogators based on this code in allcall requests. The interrogator code is also included in allcall replies to enable interrogators with overlapping ranges to separate replies from aircraft. It is important to note that the interrogator code is not included in selective roll-call replies since the design implicitly assumes that a ground sensor knows which kind of roll-call reply belongs to its preceding request.

\section{THE OPENSKY NETWORK}

The OpenSky Network is a crowd-sourced sensor network collecting air traffic control (ATC) data. Its objective is to make real-world ATC data accessible to the public and to support the development and improvement of ATC technologies and processes. Since 2012, it continuously collects air traffic surveillance data. Unlike commercial flight tracking networks (e.g., Flightradar24, FlightAware, Plane Finder), the OpenSky Network keeps the raw Mode $\mathrm{S}$ messages as they are received by the sensors in a large historical database and the data is available to researchers and analysts from different areas for free. The OpenSky sensors are of different types with some sensors collecting all Mode $\mathrm{S}$ messages while others only the ADS-B messages. For more information on OpenSky's history, architecture and use cases refer to [4], [5].

The network started out with eight sensors in Switzerland and Germany and has grown to more than 50 currently active receivers at locations all around the world. As of this writing, OpenSky's dataset contains three years of ATC communication data. While the network initially focused on ADS-B only, it extended its data range to the full Mode S downlink channel 

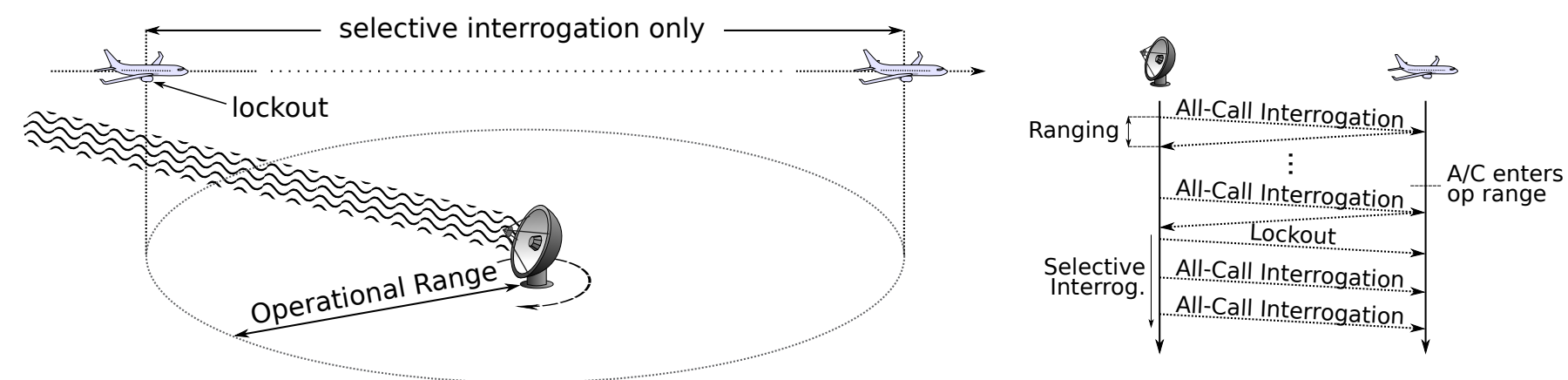

Figure 1: SSR Mode S target acquisition, selective interrogation and the lockout protocol according to [2]. New aircraft are discovered by broadcasting all-call interrogations. Once an aircraft enters the operational range of an interrogator, it tells the aircraft not to respond to future all-calls and starts interrogating the aircraft selectively.

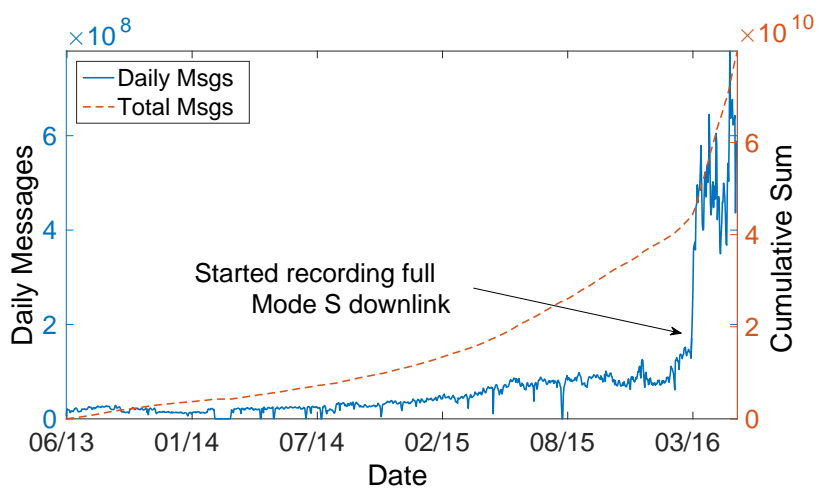

Figure 2: The growth of OpenSky's dataset over time from June 2013 to May 2016.

in March 2016. The dataset currently contains more than 150 billion Mode S or ADS-B messages from more than 80,000 transponders. The growth of the dataset is depicted in Figure 2. Besides the payload of each Mode S downlink message, OpenSky stores additional metadata. Depending on the receiver hardware, this includes precise timestamps, receiver location, and signal strength.

\section{A. Passive Monitoring Challenges}

As explained in the previous sections, Mode S, with the exception of the extended squitter, is designed for interrogationbased surveillance. This system design is challenging for passive observers such as OpenSky, which do not see the uplink communication, namely the interrogations. For example, the position of an aircraft is usually determined by the interrogator using ranging techniques (see Figure 1 ) and by the angle of the signal arrival. As a consequence, a passive observer must perform multilateration to obtain the position of all transmitting aircraft which do not provide their positions through ADSB position reports. Performing multilateration requires a high sensor density since the signal must be received by at least four receivers [4]. OpenSky, however, is not yet performing multilateration continuously and positions are therefore only determined for ADS-B equipped aircraft.

\begin{tabular}{|l|l|l|}
\hline DF: 5 bit & Payload: 27 or 83 bit & CRC $\oplus$ ID: 24 bit \\
\hline
\end{tabular}

Figure 3: The basic structure of Mode $S$ downlink replies. The downlink format (DF) determines the structure of the payload. The last 24 bit field contains the cyclic redundancy checksum XORed with either the interrogator's ID (all-call) or the transponder's ICAO 24-bit address.

Another challenging task for a passive receiver is error detection and correction in SSR. Although Mode S messages in SSR employ a cyclic redundancy check (CRC) to do so, the checksum is XORed with the aircraft's ICAO 24-bit address or the interrogator ID (all-call replies) in Mode S replies to save bits (see Figure 3). While this does not pose a problem for the interrogator since it knows which address to expect, a passive observer needs to learn the respective addresses first in order to make use of the CRC. OpenSky learns them either through all-call replies or ADS-B messages, through the reception of multiple messages with the same address within a short amount of time, or by receiving the identical message with multiple receivers. Once an ICAO 24-bit address has been accepted, it is added to a "currently tracked" list and all messages with this ID will be accepted. An address is purged from the list after no message has been received from this transponder for a certain amount of time. For all-call replies, we can check the validity of the interrogator ID: There are only 80 valid interrogator IDs out of the $2^{24} \approx 16.8$ million possible. Thus, there is only a small possibility that a faulty packet contains a valid interrogator ID. As a result, all-call replies (DF 11) can be checked for validity with a reasonable false positive rate.

Decoding Comm-B messages is further challenging since they do not have a fixed message type field that allows for uniquely identifying the content of the message. Once again, this does not pose a problem for the interrogator since it knows which data it requested. In OpenSky, a variety of plausibility checks on the data fields and message structure need to be performed in order to determine their content. This, however, is out of the scope of this paper and subject to future work. 


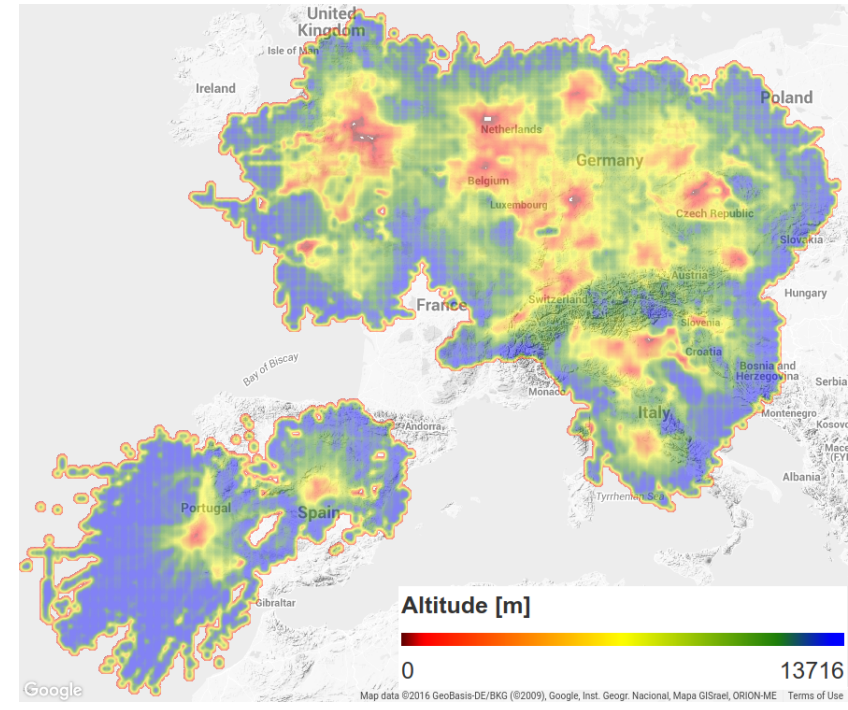

Figure 4: The three-dimensional coverage of the OpenSky Network in Europe as of 2016/05/23. The overall coverage is divided in six unconnected parts in Europe, North America, and New Zealand. The total area covered in the en route airspace is about $\mathbf{3 . 4}$ million square kilometers.

\section{DATA ANALYSis}

This section provides statistics on SSR Mode S and ADS-B usage based on data from the OpenSky Network. In order to help understanding and interpreting our results correctly, we first clarify the quality and the limitations of our data. Then, we present our results on Mode $S$ downlink formats frequency analysis, ADS-B deployment status, ADS-B standard conformance, and ADS-B position accuracy.

\section{A. Data Quality \& Limitations}

The most natural limit of our data is OpenSky's coverage. The OpenSky Network currently only covers parts of Europe, America, and New Zealand. Hence, our analysis explicitly does not cover or represent the situation in the Asian, Australian, South American, or African airspaces. It rather constitutes a snapshot of the situation mainly in Europe and parts of the USA. Since receiving Mode S and ADS-B signals requires a line of sight between receiver and aircraft, the ranges of receivers are limited by the radio horizon. For example, if the aircraft is in the en-route airspace, i.e. at a high altitude, and the receiver is not obstructed by the geographical environment (e.g., in coastal areas), the radio horizon and thus the range can be up to $700 \mathrm{~km}$. Aircraft at lower altitudes, however, remain difficult to track due to their reduced line of sight. As a consequence, lower altitudes are only covered if there is a sensor nearby. Figure 4 shows the three-dimensional coverage over Europe. The European coverage sums up to a total of about three million $\mathrm{km}^{2}$. The information about the US airspace is derived from data from two sensors in the Los Angeles and Washington area with a total coverage of about $170.000 \mathrm{~km}^{2}$. In addition, the dataset contains data from one sensor in New Zealand with a total coverage of $200.000 \mathrm{~km}^{2}$.

\begin{tabular}{|l|l|l|}
\hline Percentage & Type & DF \\
\hline \hline $32 \%$ & all-call replies & 11 \\
\hline $26 \%$ & ADS-B messages & 17 \\
\hline $15 \%$ & altitude reply (ACAS) & 0 \\
\hline $10 \%$ & altitude reply, Comm-B & 20 \\
\hline $9 \%$ & altitude reply & 4 \\
\hline $4 \%$ & squawk, Comm-B & 21 \\
\hline $2 \%$ & squawk & 5 \\
\hline $1 \%$ & altitude, threat resolution (ACAS) & 16 \\
\hline$<1 \%$ & ADS-B/TIS-B message (non-transponder) & 18 \\
\hline$<1 \%$ & ELM, Comm-D & 24 \\
\hline $0 \%$ & Military applications & 19 \\
\hline
\end{tabular}

Table II: Mode S downlink formats, ordered by the percentage of the respective DF in OpenSky's data.

Another limiting factor is the lack of publicly accessible meta information about aircraft. For example, there is no official, publicly accessible, database which links the ICAO 24-bit addresses with meta-information that can be used to, for instance, identify unmanned aerial vehicles (UAV) or the owner of aircraft. There are public data sources that allow a limited analysis and categorization of aircraft (see Section $\mathrm{V}$-A . These sources are, however, mostly compiled by hobbyists and there are no guarantees for their correctness and completeness. In fact, during our research we found that, e.g., non-commercial aircraft with Spanish registrations were entirely missing from the sources available to us. It is worth noting that maintaining such datasets is challenging since many different official sources and information from live trackers need to be joined and continuously updated.

The most important limitation is the data quality. For example, ADS-B is still in its deployment phase and there are no guarantees that transponders are functioning according to the specification. In fact, we found that a small number of transponders broadcast erroneous or invalid positions, or wrong ICAO 24-bit addresses. Furthermore, receivers as well are not certified. Due to missing implementations of proper tracking techniques, erroneous messages can pass the error detection mechanism of Mode S and therefore end up in our data. Although we have a multitude of plausibility checks running to filter most of these invalid data, a small amount may still remain in the data used for this work. Nevertheless, based on our experience from working with Mode S and ADS-B for many years, we are confident that the portion of erroneous data is negligible compared to the overall size of the dataset and that the numbers provided in this work are accurate estimates of the situation in OpenSky's coverage area.

Finally, it is worth mentioning that the dataset also includes ADS-B messages from surface vehicles. These transponders oftentimes are ADS-B only transponders and may bias our statistics towards ADS-B. However, since ADS-B has considerably lower message rates on ground and given that our ground coverage is extremely limited, we believe that the fraction of messages from ground vehicles is negligibly small. 


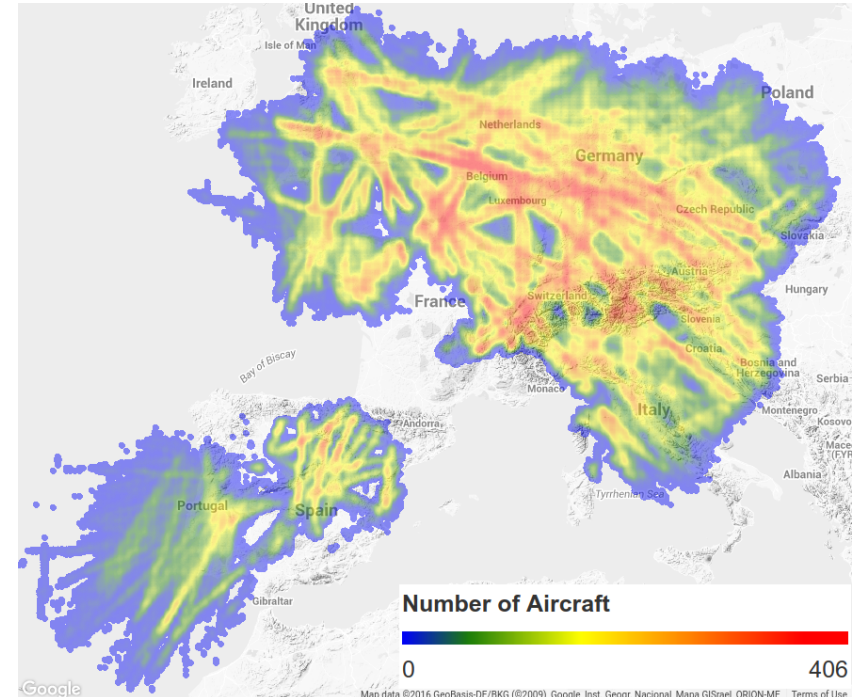

Figure 5: The heatmap shows the number of aircraft seen during one day per position for Europe. Heavily used routes connect the major European airports such as Brussels, Frankfurt, London, Paris, Prague, and Zurich.

\section{B. The Dataset \& Received Downlink Formats}

The dataset used to calculate the statistics below contains all Mode S downlink messages received between 2016/04/01 and 2016/05/04 (34 days). The percentages of the different downlink formats are shown in Table II The most common downlink format was 11 (all-call replies) with a percentage of $32 \%$, followed by ADS-B with $26 \%$. However, in total, altitude replies dominate the dataset with a total percentage of $35 \%$ consisting of air-to-ground altitude replies (DF 4+20) and airto-air altitude replies (DF 0+16). Identification replies (DF 5, 21) amount only to $6 \%$ of the dataset. ADS-B messages from non-Mode $S$ transponders (e.g., used by some airport vehicles) as well as Comm-D datalink messages are both less than $1 \%$. The undocumented format reserved for military applications (DF 19) was practically non-existent.

\section{C. $A D S-B$}

Figure 5 shows a heatmap of Europe with the number of aircraft that reported ADS-B position messages on the 2016/05/23. The routes between European capitals and traffic hubs such as London and Frankfurt are clearly visible.

Figure 6 shows the number of distinct transponder IDs that were seen by OpenSky between 2016/04/01 and 2016/05/04 . We categorized them by continent based on their ICAO 24bit ID and assumed a transponder being ADS-B equipped if it transmitted DF 17 messages. One artifact observed in Figure 6 is that the percentages of ADS-B transponders are much higher for continents which are not covered by the OpenSky Network (i.e., Asia, Africa, and South America). A reason could be that aircraft carrying out transcontinental flights have been upgraded earlier in order to meet all the diverse regulations across various ANSPs. Another aspect worth noting is that

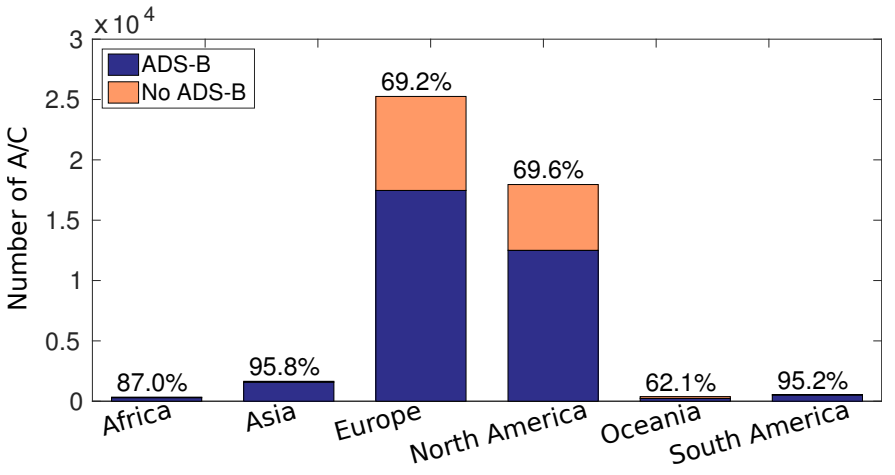

Figure 6: Origin and ADS-B equipage of Mode S-equipped aircraft seen in OpenSky's coverage between 2016/04/01 and 2016/05/04. Note that the numbers for Africa, Asia, and South America do not reflect the situation in their domestic airspaces. For continents covered by the OpenSky Network we conclude that about $70 \%$ of all Mode $S$ transponders have ADS-B capabilities.

these statistics include military aircraft. Since most military aircraft do not broadcast ADS-B messages, they lower the percentage slightly since they are still responding to Mode $S$ interrogations. Especially for continents with only few known aircraft (e.g., Africa), the numbers may not properly represent the equipage of airliners.

In addition, it is important to know that these numbers do not include aircraft which are not equipped with Mode $S$ transponders. These aircraft are not detected by the OpenSky Network at all. For example, general aviation flying under visual flight rules only are not necessarily be equipped with Mode S transponders. However, we conclude that from OpenSky's perspective, around 70\% of all Mode S-equipped aircraft in Europe and the US are equipped with ADS-B Out.

Standard Conformance: We find that most of the ADS-B transponders do not yet fully implement the ADS-B specification DO-260B [6]. When airborne, the specification requires ADS-B transponders to automatically broadcast at least position, identification, and velocity information. Operational status messages are only broadcast if the respective information are available and valid. Event-driven message types such as emergency and priority status messages are only broadcast for the duration of the event. As Table [III shows, $72.94 \%$ of all transponders broadcast at least one type of ADS-B messages. While position and velocity are broadcast by most of these transponders $(85.16 \%)$, only $54 \%$ broadcast identification messages (callsign and aircraft category). Overall, only $41.28 \%$ of ADS-B transponders broadcast all information required by the standard.

It is worth noting that those $58.72 \%$ of ADS-B transponders which do not transmit all information mostly seem to omit identity messages while position reports are omitted by only about $11 \%$. The numbers in Table III suggest that aircraft either transmit both position and velocity or none of 


\begin{tabular}{|c|c|c||c|c|}
\hline Position & Velocity & Identification & \% (ADS-B only) & $\%$ (all) \\
\hline \hline $\boldsymbol{X}$ & $\boldsymbol{X}$ & $\boldsymbol{X}$ & - & 27.04 \\
$\boldsymbol{X}$ & $\boldsymbol{X}$ & $\boldsymbol{J}$ & 10.99 & 8.02 \\
$\boldsymbol{X}$ & $\boldsymbol{J}$ & $\boldsymbol{X}$ & 0.17 & 0.12 \\
$\boldsymbol{X}$ & $\boldsymbol{J}$ & $\boldsymbol{J}$ & 0.37 & 0.27 \\
$\boldsymbol{J}$ & $\boldsymbol{X}$ & $\boldsymbol{X}$ & 1.94 & 1.42 \\
$\boldsymbol{J}$ & $\boldsymbol{X}$ & $\boldsymbol{J}$ & 1.36 & 0.99 \\
$\boldsymbol{J}$ & $\boldsymbol{J}$ & $\boldsymbol{X}$ & 43.88 & 32.01 \\
$\boldsymbol{J}$ & $\boldsymbol{J}$ & $\boldsymbol{J}$ & 41.28 & 30.12 \\
\hline
\end{tabular}

Table III: Percentage of aircraft broadcasting a certain combination of ADS-B messages. Overall, $72.96 \%$ transmitted at least one message type.

\begin{tabular}{|c|l|l|}
\hline SIL & Probability of Exceeding the HCR & Percentage of A/C \\
\hline \hline 0 & unknown & $24.77 \%$ \\
\hline 1 & $10^{-3}$ per flight hour or operation & $2.89 \%$ \\
\hline 2 & $10^{-5}$ per flight hour or operation & $23.50 \%$ \\
\hline 3 & $10^{-7}$ per flight hour or operation & $46.83 \%$ \\
\hline
\end{tabular}

Table IV: Surveillance Integrity Level (SIL) reported by aircraft. It indicates the probability of exceeding the horizontal containment radius (see Figure 7 ) and only depends on the quality of the position sensor used.

them. By checking a random sample of aircraft that do not broadcast their position with public databases (see Section $\mathrm{V}-\mathrm{A}$, we found that most of them are either military aircraft or small aircraft flying under visual flight rules such as gliders.

Operational Status: The operational status of an aircraft includes information about the aircraft such as ADS-B version, traffic alert and collision avoidance system (TCAS) availability, ADS-B In availability, use of multiple antennae or position accuracy information. This information, however, is only broadcast by an aircraft if the transponder supports it. In total, we found that only $21 \%$ of the aircraft equipped with ADS-B broadcast operational status reports. Of the $21 \%$, only $0.16 \%$ report ADS-B version 0. Most aircraft use ADS-B version 1 (46.14\%) and version 2 (53.70\%). Information about TCAS has been in the standards since version 0 and $45.3 \%$ of the aircraft report having an operational TCAS. Since ADS-B version 2, aircraft also report whether they have the capability to receive ADS-B reports from other aircraft. Out of those aircraft reporting ADS-B version 2, $16.62 \%$ aircraft report having either 1090ES or Universal Access Transceiver (UAT) In function. The UAT datalink has been selected by the U.S. for general aviation that are not capable of high altitudes [7]. In contrast, 1090ES has been selected as the globally interoperable ADS-B datalink for commercial aviation [8]. However, slightly more aircraft report having UAT In (10.76\%) than 1090ES In $(9.49 \%)$. Only $3.63 \%$ support both functions at the same time.

Position Accuracy: We find that almost all aircraft (99.94\%) use barometric altitude rather than global navigation satellite system (GNSS)-derived height in their position messages. Transponders with GNSS time synchronization can synchronize their transmitting subsystem with the position

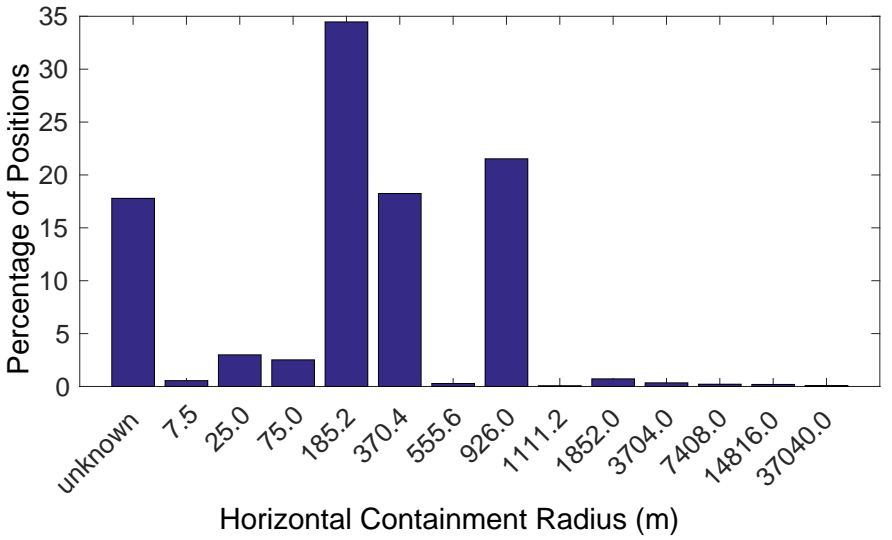

Figure 7: Distribution of reported horizontal containment radii. The HCR are determined using the navigation integrity category field of each airborne position message.

source. In this mode, the transponder interpolates the position to compensate for the delay between the time when the position is determined and the time when it is broadcast to further improve the position accuracy. However, only $3.5 \%$ of all aircraft indicate the possibility of GNSS-timed transmission of position reports. The accuracy of the position reported by ADS-B is indicated by the navigation integrity category (NIC, [9, 2.1.2.12]) in position reports in conjunction with the source integrity level (SIL, [9, 2.1.2.15]) in operational status messages. The NIC determines the horizontal containment radius (HCR) and the SIL the probability for the actual position to lie within this radius. It is worth noting that the SIL only depends on the on-board sensor used to determine the position. For example, if there are temporarily not enough satellites available for a position fix, the NIC reported becomes 0 (unknown) but the SIL remains unchanged. The distribution of the HCR reported by aircraft is shown in Figure 7 . In addition, the percentages of aircraft reporting a certain SIL is shown in Table IV About $80.59 \%$ of all positions were reported with an HCR below $1 \mathrm{~km}$. The residual position reports consist of $17.79 \%$ with unknown radii and $1.63 \%$ with a radius above $1 \mathrm{~km}$.

It is worth noting that a containment radius of $1 \mathrm{~km}$ appears large at first but given the hard guarantees associated with it (see Table IV), it can be considered as a relatively pessimistic bound. To get a more realistic position accuracy estimate, we additionally considered the navigation accuracy category (NAC) of the navigation information used as the basis for the aircraft reported positions. It is contained in operational status reports, so we only have this information for about $21 \%$ of all ADS-B equipped aircraft (see above). The NAC determines the so called estimated position uncertainty (EPU) ${ }^{3}$ The EPU is the $95 \%$ accuracy bound for the position fix, i.e., the probability of the distance between the reported and actual position being greater than this bound is 0.05 . According to

\footnotetext{
${ }^{3}$ In conjunction with GNSS systems such as GPS, the EPU is also called horizontal figure of merit.
} 


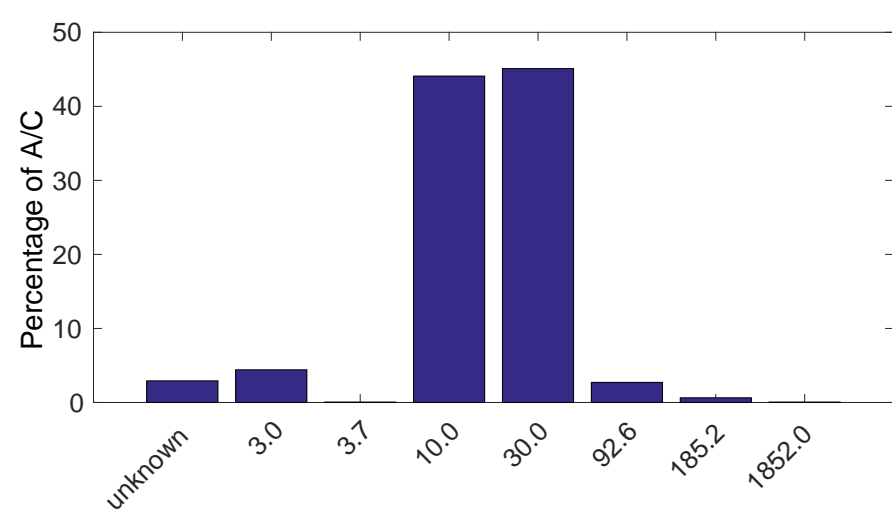

Estimated Position Uncertainty (m)

Figure 8: Distribution of reported estimated position uncertainties (EPU). They provide an upper bound for the error of $95 \%$ of the reported positions. The EPU has been determined using the navigation accuracy category field of operational status messages.

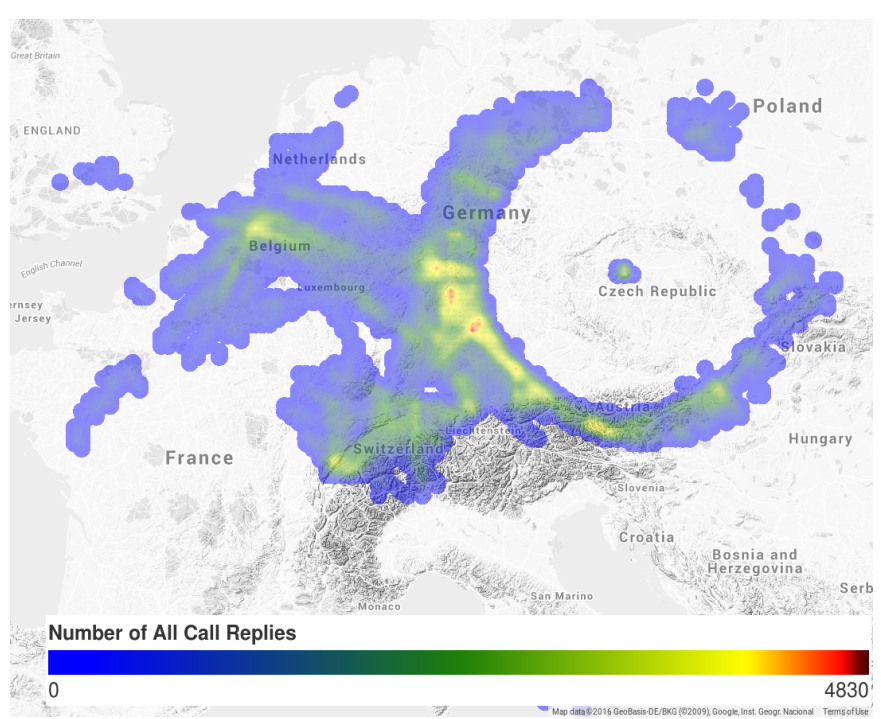

Figure 9: Heatmap showing the density of all-call replies for interrogators with ID 0x1a. The circle around Václav Havel Airport in Prague demonstrates the effect of the Mode S lockout protocol. The operational range of the Prague radar (radius of the circle) is about $277 \mathrm{~km}$ (150 nautical miles).

the results shown in Figure 8, the EPU is reported to be at most $30 \mathrm{~m}$ in $93.66 \%$ of the status messages. We can thus conclude that the error of positions reported by ADS-B is at most $30 \mathrm{~m}$ in about $89 \%$ of the time.

\section{Interrogation Patterns}

Figure 9 shows the effect of the lockout protocol of a radar at Václav Havel Airport in Prague (interrogator ID 0x1a). The operational range of the radar is about 150 nautical miles (277 $\mathrm{km})$. As indicated by the blue dot in the middle of the circle in Figure 9, Mode $\mathrm{S}$ does not work for positions very close the

\begin{tabular}{|l|c|c|}
\hline Aircraft Affiliation & Aircraft & {$[\mathbf{\%}]$} \\
\hline Private/Business & 9,370 & $24.6 \%$ \\
\hline Military & 1,125 & $3.0 \%$ \\
\hline State-related & 213 & $0.1 \%$ \\
\hline Scheduled/Other & 20,623 & $54.2 \%$ \\
\hline Ground/Airport & 376 & $0.1 \%$ \\
\hline \hline Unidentified & 6,006 & $15.8 \%$ \\
\hline
\end{tabular}

Table V: Types of owners of 38,073 ADS-B equipped aircraft seen by the OpenSky Network. They were identified by comparing the ICAO 24-bit transponder IDs with public sources.

the interrogator. This effect is known as the "cone of silence". The elevation angle of the beam of the directed ground antenna does usually not allow for purely vertical interrogations. The result is a cone-shaped area above the ground antenna in which the interrogator lacks coverage.

\section{IDENTIFYING AIRCRAFT}

By incorporating additional public data sources, we classify the aircraft seen by OpenSky regarding their ownership.

\section{A. Sources of Aircraft Metadata}

There are several public data sources available that provide meta information on aircraft based on their identifiers (ICAO 24 bit identifier or callsign). This information typically includes the aircraft type (e.g., Airbus A320) and the owner/operator (e.g., British Airways), which can be exploited for further in-depth analysis. The public databases can broadly be divided into online and offline sources.

Online sources are websites with query possibilities returning meta information and, in the case of flight tracking websites, the movement of aircraft. For official information, some countries' aviation authorities (e.g., US, UK, Australia) offer interactive database lookups on their websites. However, these are naturally limited to all aircraft registered in a given country and cannot provide a centralized source. The not-forprofit project Airframes.org is a highly valuable site for learning general aircraft information as it offers very comprehensive data, including background knowledge such as pictures and historical ownership information [10]. Lastly, commercial projects such as Flightradar24 offer both aircraft metadata and flight tracking, i.e. historical movement data [11].

Offline sources are aircraft databases, typically in SQLite or CSV format provided by third parties. We use two such sources for our analysis of metadata. The first database is available and constantly updated in the Planeplotter software [12]. We use a version of the SQLite database file created in November 2014 containing 120,149 rows of aircraft data. The second database, known as the World Aircraft Database has been collecting aircraft information over a period of 8 months at the time of writing, amounting to 89,391 rows [13].

\section{B. Aircraft Identification}

Identification of aircraft is limited to those contained in the public data sources. There are two major interest groups which 
seek out blocks from internet databases: state actors such as air forces and government aircraft, and privately-owned aircraft be it by companies, institutions, or individuals.

We used the above on- and offline sources to identify 38,073 ADS-B transponders seen in April 2016. The data indicates that $84.2 \%$ of all transponders are identifiable via public data sources. Table $\mathrm{V}$ breaks down the aircraft types by their affiliation. The type is determined by the registrar and the type of the aircraft, i.e., if a non-airline business is the registrar then it is classed as a business aircraft.

Unmanned Aerial Vehicles: Besides manned aircraft, we have also started to encounter UAVs within OpenSky. Naturally, as UAVs are poised to enter the civil airspace, some utilize ADS-B to be able to move safely and cooperate with air traffic control. Consequently, they are visible to flight trackers and ADS-B/Mode S sensors in general. We analysed the historical data of OpenSky based on our publicly available aircraft information to see whether we could find evidence of Mode $\mathrm{S}$ and ADS-B-equipped UAVs.

Currently, the only such evidence is of UAVs used by the Swiss Airforce, which are publicly traceable on commercial websites and also with OpenSky [14]. These UAVs, called 'ADS 95 Ranger Drones', use Mode S to communicate their identification and altitude and can subsequently be localised using multilateration by interested flight trackers.

Unfortunately, we could not identify further UAVs in an automated fashion as the ICAO has not assigned an aircraft type designator to drones, preventing them from being widely included in our public databases ${ }^{4}$ Whether more UAVs will be registered with the civil aviation authorities and issued their own 24-bit addresses will remain to be seen in the future. Apart from this straight-forward detection approach, we consider researching detection methods for UAVs based on their specific behavior (e.g., extremely high cruising altitudes, very slow speeds, or flight endurance) in the future.

\section{Deployment Plans \& Status}

While SSR Mode $\mathrm{S}$ has become operative in parts of the world (e.g. Europe) in the 2000s, ADS-B is still in its deployment phase. Figure 10 illustrates the mandate timeline for ADS-B equipage in Europe and the US. Through CFR 91.225 issued in June 2010, the FAA set the ADS-B Out equipage deadline for $1^{\text {st }}$ January 2020 on all aircraft with an electrical system in designated airspace [15]. In Europe, the European Commission issued Regulation 1207/2011 mandating the Single European Sky [16]. As part of this, ADS-B Out usage is mandated on aircraft built after $8^{\text {th }}$ January 2015, and for all aircraft by $7^{\text {th }}$ December 2017. Regulation 1028/2014 later modified this to bring it in line with FAA efforts, pushing the deadline back to $1^{\text {st }}$ January 2020 [17].

ADS-B forms a key part of the Global Air Navigation Plan (GANP) 2013 produced by ICAO [18]. It indicates ADS-B being used from 2013 for surveillance purposes, with surface

\footnotetext{
${ }^{4}$ Currently, the ADS 95 Ranger and any other UAV would use the unassigned code 'ZZZZ'.
}

surveillance from 2018. In the longer term, the GANP implies the creation and deployment of a 'future' ADS-B.

As of February 2016, FAA statistics indicate that 16,765 general aviation (GA) aircraft and 451 aircraft of air carriers (i.e. aircraft belonging to commercial airlines) are ADS-B equipped [19]. Comparing this to the newest publicly available US Department of Transportation inventory statistics from 2014 [20], currently $6.8 \%$ of US carrier aircraft and $8.2 \%$ of general aviation are equipped. This is reinforced by similar figures stated in [21], which predicts that at current rates, commercial aircraft will be equipped in time. However, in both cases, this falls well short of the FAA goals for general and carrier aviation. For GA, current equipage is approximately half of the number needed to hit 100,000 by 2020 . This is similar for carriers, with current levels under half the required amount for 5,000 aircraft equipped by 2020. More recently, the FAA states that over 21,000 aircraft are ADS-B Out equipped [22].

Official equipage statistics for aircraft elsewhere in the world are currently limited and to the best of our knowledge, no public statistics exist for Europe. For Air Navigation Service Providers, who are responsible for air traffic control, ADS-B deployment seems varied with many using it side-byside with traditional methods. For example, in reference to a trial of ADS-B for GA, the National Air Traffic Service (NATS) of the UK note that they do not rely on ADS-B [23].

\section{A. Comparing the Official Numbers to OpenSky's Data}

Comparing the official numbers on ADS-B equipage to that derived by OpenSky's dataset is difficult for several reasons. OpenSky only considers the SSR Mode S downlink and therefore only detects 1090ES-based ADS-B transponders. We cannot draw any conclusions on the UAT-based ADS-B deployment which, for example, covers most of the U.S. general aviation. In addition, public sources of information on the current ADS-B deployment mostly lack important information on how the numbers are derived, which transponders exactly are covered by the numbers, and at which level transponders are considered having ADS-B capability.

The numbers provided in this work consider transponders having ADS-B capability as soon as they broadcast extended squitters containing valid ADS-B messages. In particular, they do not reflect the percentage of aircraft having certified and complete $A D S-B$ capabilities.

\section{Future Plans}

The OpenSky Network is an ongoing effort that improves and extends its capabilities continuously. As of this writing, new sensors are being deployed around the world and existing sensors from the community become integrated. Apart from the ability to monitor, e.g., the progress of ADS-B deployment or Mode $\mathrm{S}$ usage, growth and increased receiver density will allow us to perform multilateration to additionally locate non-ADS-B aircraft. In addition, other sensors for wireless ATC technologies such as the surveillance protocols FLARM and UAT ADS-B or the general purpose datalinks ACARS 


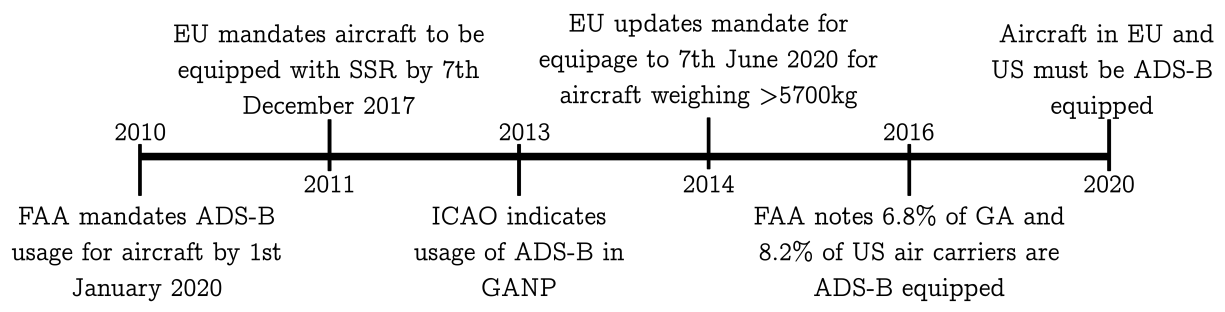

Figure 10: ADS-B mandate and deployment timeline.

and CPDLC are planned to be included in the OpenSky Network to provide a more complete picture of the global airspace. Besides integrating new technologies, we also aim at incorporating additional sources of metadata such as aircraft databases or weather data. Merging this metadata with the data provided by the sensors widens the range of possible applications and analyses in OpenSky considerably.

These goals, however, require the support of the community for sensor sponsoring and hosting and the willingness to share data. To join and support the OpenSky Network initiative, please refer to http://opensky-network.org.

\section{CONCLUSION}

In this paper, we analyzed the usage of Mode S and ADS-B in large areas in Europe, North America, and New Zealand. The data used for this analysis is collected by the OpenSky Network, a research initiative which continuously monitors and records air traffic control communications using more than 50 Mode $\mathrm{S}$ sensors across the globe. Based on our analysis, we can conclude that about $70 \%$ of all Mode $\mathrm{S}$ transponders in Europe and the United States of America are already upgraded with ADS-B capabilities. We further provided insights on the composition of Mode $\mathrm{S}$ downlink communications, the current state of ADS-B standard conformance in aircraft transponders, and the quality and accuracy of their position reports. Lastly, we identified and categorized the aircraft tracked by OpenSky by incorporating and comparing publicly available data sources.

\section{ACKNOWLEDGMENTS}

The authors would like to thank Andrea Schlapbach from FLARM Technology for his valuable comments and important suggestions that helped improve this paper.

\section{REFERENCES}

[1] International Standards and Recommended Practices, Annex 10: Aeronautical Telecommunications, 4th ed., International Civil Aviation Organization (ICAO), 2007, Volume IV: Surveillance and Collision Avoidance Systems.

[2] EUROCONTROL, "Principles of Mode S Operation and Interrogator Codes," Mar. 2003

[3] International Standards and Recommended Practices, Annex 10: Aeronautical Telecommunications, 2nd ed., International Civil Aviation Organization (ICAO), 2007, Volume III: Communication Systems.

[4] M. Schäfer, M. Strohmeier, V. Lenders, I. Martinovic, and M. Wilhelm, "Bringing Up OpenSky: A Large-scale ADS-B Sensor Network for Research," in Proceedings of the 13th IEEE/ACM International Symposium on Information Processing in Sensor Networks (IPSN), Apr. 2014.
[5] M. Strohmeier, M. Schäfer, M. Fuchs, V. Lenders, and I. Martinovic, "Opensky: A swiss army knife for air traffic security research," in Proceedings of the 34th IEEE/AIAA Digital Avionics Systems Conference (DASC), Sep. 2015

[6] RTCA Inc., "Minimum Operational Performance Standards for 1090 MHz Extended Squitter Automatic Dependent Surveillance - Broadcast (ADS-B) and Traffic Information Services - Broadcast (TIS-B)," DO260B with Corrigendum 1, Dec. 2011.

[7] J. Scardina, "Overview of the FAA ADS-B Link Decision," Federal Aviation Administration, Jun. 2002.

[8] C. Spitzer, U. Ferrell, and T. Ferrell, Digital Avionics Handbook, 3rd ed. CRC Press, Sep. 2014.

[9] RTCA Inc., "Minimum Aviation System Performance Standards for Automatic Dependent Surveillance Broadcast (ADS-B)," DO-242A, Jun. 2002.

[10] R. D. Kloth, “Airframes.org," 2016. [Online]. Available: http: //www.airframes.org/

[11] FlightRadar24 AB, "Flightradar24," 2016. [Online]. Available: https: //www.flightradar24.com

[12] Centro de Observação Astronómica no Algarve, "PlanePlotter," 2016. [Online]. Available: http://www.coaa.co.uk/planeplotter.htm

[13] J. Sun, "World Aircraft Database," 2016. [Online]. Available: http://junzisun.com/adb/

[14] M. Strohmeier, M. Smith, M. Schäfer, V. Lenders, and I. Martinovic, "Assessing the impact of aviation security on cyber power," in Cyber Conflict (CYCON), 2016 8th International Conference on. IEEE, 2016.

[15] Federal Aviation Administration, "14 CFR Part 91 Automatic Dependent Surveillance- Broadcast (ADS-B) Out Performance Requirements To Support Air Traffic Control (ATC) Service; Final Rule," pp. 1-37, 2010.

[16] The European Commission, "Commission regulation laying down common airspace usage requirements and operating procedures for airborne collision avoidance," pp. 2008-2010, 2011.

[17] — , "Implementing Regulation (EU) No. 1028/2014 amending Implementing Regulation (EU) No 1207/2011,” pp. 8-9, 2014.

[18] International Civil Aviation Organization, "Global Air Navigation Plan Fourth Edition," International Civil Aviation Organization, Montreal, Tech. Rep., 2013. [Online]. Available: http://www.icao.int/publications/ Documents/9750_4ed_en.pdf

[19] Federal Aviation Administration, "Equip 2020 FAA Update," Presentation, Atlanta, feb 2016. [Online]. Available: http://www.rtca.org/Files/ CommitteeMeetingSummaries/NACmtgpptv5fnlActionsIncluded.pdf

[20] Untied States Department of Transportation: Bureau of Transportation Statistics, "Table 1-11: Number of U.S. Aircraft, Vehicles, Vessels, and Other Conveyances," 2014. [Online]. Available: http://www.rita.dot.gov/bts/sites/rita.dot.gov.bts/files/publications/ national_transportation_statistics/html/table_01_11.html

[21] W. Bellamy III, "ADS-B Case Study: Equipping 100,000 Aircraft," Avionics Magazine, jun 2016. [Online]. Available: http://www. aviationtoday.com/av/issue/feature/87845.html

[22] Federal Aviation Administration, "Automatic Dependent SurveillanceBroadcast," 2016. [Online]. Available: https://www.faa.gov/nextgen/ update/progress_and_plans/adsb/

[23] R. Hayward, "Helping general aviation to see and be seen in our busy airspace," 2016. [Online]. Available: http://nats.aero/blog/2016/03/ helping-general-aviation-to-see-and-be-seen-in-our-busy-airspace/ 